\title{
Applications of plasmonics: general discussion
}

Vladimir Bochenkov, Jeremy Baumberg, Mikhail Noginov, Felix Benz, Hasan Aldewachi, Silvan Schmid, Viktor Podolskiy, Javier Aizpurua, Kaiqiang Lin, Thomas Ebbesen, Alexei A Kornyshev, James Hutchison, Katarzyna Matczyszyn, Samir Kumar, Bart de Nijs, Francisco Rodríguez Fortuño, James T. Hugall, Pablo de Roque, Niek van Hulst, Santhosh Kotni, Olivier Martin, F. Javier García de Abajo, Michael Flatté, Andrew Mount, Martin Moskovits, Pavel Ginzburg, David Zueco, Anatoly Zayats, Sang-Hyun Oh, Yu Chen, David Richards, Alessandro Belardini and Prineha Narang

DOI: $10.1039 / c 5 f d 90025 e$

F. Javier García de Abajo opened a general discussion of the paper by David Zueco: How do you interpret inelastic scattering in the ultrastrong coupling limit? Is part of the energy remaining in the system, while a photon with lower energy is emitted?

David Zueco responded: It is a Raman scattering process. The photon interacts with the hybrid (because they are ultrastrongly coupled) qubit-waveguide. After the interaction, the photon emitted has less energy. This energy stays in an excited state of the hybrid qubit-waveguide. We have checked this picture in our numerical calculations.

Jeremy Baumberg asked: Can you label clearly the curves of your energies shown, and show how they map onto $\mathrm{P}+$ and $\mathrm{P}-$ at low $g$ ? Also, can you track how the excited final state that a single photon scattering off the system leaves changes as the coupling decreases from the ultrastrong regime? It is very unclear how to map your model of the inelastic scattering onto something involving the original strong coupling states. Can you trace out this in a figure, as it would be very useful. When one photon goes in, and comes out at a different energy, then there must be some energy left in the system, but where is it? Surely we must conserve energy in the system, but how does the lack of conservation of excitations work. Are you just saying that we have some sort of collective mode forming (like a plasmon)?

David Zueco replied: Thanks a lot for your interest. When a photon impinges on a qubit (or more) in the ultrastrong coupling regime we see that we have got 
inelastic scattering, provided that $g$ and $\hbar \omega_{\text {in }}$ are large enough. In that case, a new photon emerges with a different energy. We show the probability of having this inelastic scattering in Fig. 1 as a function of input energy $h \omega_{\text {in }}$ and coupling constant $g$. The output is a flying photon with different energy $\hbar \omega_{\text {out }}$ and the system does not come back to the ground state, but we find that instead it ends up in a dressed bound state $\left|E_{2}\right\rangle$. The energy of this new photon ( $\hbar \omega_{\text {out }}$ ) will be that of the incident one $\left(\hbar \omega_{\text {in }}\right)$ minus the gap between the excited and the ground state $|\mathrm{GS}\rangle$ :

$$
\hbar \omega_{\text {out }}=\hbar \omega_{\text {in }}-\left(E_{2}-E_{\mathrm{GS}}\right) .
$$

Because of this condition, the input energy has got a lower bound:

$$
\hbar \omega_{\text {in }} \geq \hbar \omega_{k=0}+\left(E_{2}-E_{\mathrm{GS}}\right)
$$

This equation marks a boundary for existence of this process. The dotted white line in Fig. 1 renders this boundary using the computed $E_{2}(g)$, showing the involvement of $\left|E_{2}\right\rangle$ in the Raman process.

Let us now analyse the origin and properties of $\left|E_{2}\right\rangle$. Our Hamiltonian preserves parity $P=\mathrm{e}^{i \pi N}$ :

$$
[P, H]=0,
$$

with $N$ the number operator. As the ground state is even $(P|\mathrm{GS}\rangle=|\mathrm{GS}\rangle)$, the input state is odd, so the output state is odd too. As this is a photon flying over $\left|E_{2}\right\rangle$, then $\left|E_{2}\right\rangle$ is even. The connection of $\left|E_{2}\right\rangle$ with known results is easier in the low $g$ regime, where the Rotating Wave Approximation is valid and not only the parity but also the number of particles is well defined.

A known result in this case is that in the one-excitation sector, there are two bound states $\left|\Psi_{ \pm}\right\rangle$, appearing below (-) and over (+) the band, with energies moving away from the band edges as $g$ increases (see inset to Fig. 2). These states can be analytically computed (Longo et al., ref. 2), having the expression:

$$
\left|\Psi_{ \pm}\right\rangle=a_{ \pm}^{\dagger}|\mathrm{GS}\rangle \quad a_{ \pm}^{\dagger}=\sum_{x} \phi_{x, \pm} a_{x}^{\dagger}+d_{ \pm} \sigma^{+}
$$

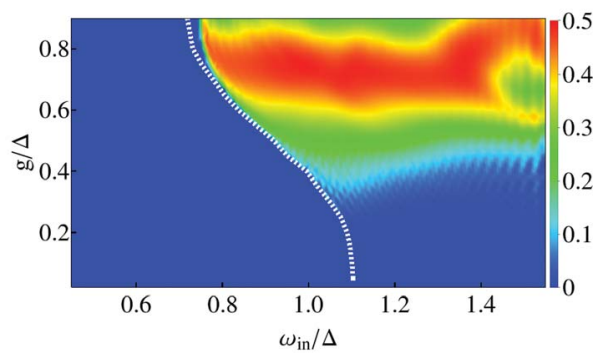

Fig. 1 Inelastic scattering. Probability of inelastic scattering in the full model as a function of both incident photon frequency $\omega_{\text {in }}$ and $g$. The white dotted line is the estimated boundary for the region where the photon frequency conversion occurs. Figure adapted from ref. 1. 


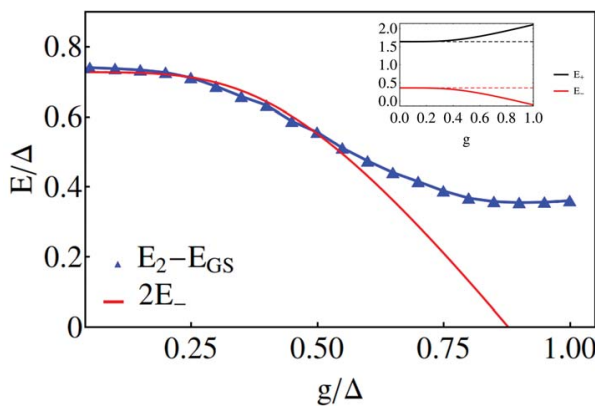

Fig. $2 E_{2}-E_{\mathrm{GS}}$ vs. $2 E_{-}$. Gap in the ultrastrong coupling $E_{2}-E_{\mathrm{GS}}$ (blue triangles) and $2 E_{-}$ (solid red curve). As seen, they are almost equal for small $g$. We show the energies of the bound states in the Rotating Wave Approximation in the single-particle sector in the inset.

These states have got one excitation and odd parity. Then, they cannot be the state involved in the Raman scattering $\left|E_{2}\right\rangle$. However, we can consider states with two excitations, e.g. $\left(a^{\dagger}\right)^{2}|\mathrm{GS}\rangle$. It is still an eigenstate in the low coupling regime, because in such a case $d_{-}$goes to zero, so $\left|\Psi_{-}\right\rangle$is almost a bosonic excitation. Fig. 2 shows that the computed $E_{2}(g)$ is, at small $g$, in excellent agreement with $2 E_{-}$, showing that $\left|E_{2}\right\rangle \approx\left(a_{-}^{\dagger}\right)^{2}|\mathrm{GS}\rangle$, something that is further confirmed by the analysis of the populations of both qubit and surrounding cavities.

Summarising, we have traced back the state involved in that inelastic scattering to an eigenstate of a qubit coupled to an array of coupled cavities in the low coupling regime. It is the ground state in the two particle sector if $g$ is small enough, or the first excited bound state with even parity for all $g$.

1 E. Sánchez-Burillo, D. Zueco, J. J. García-Ripoll and L. Martin-Moreno, Phys. Rev. Lett., 2014, 113, 263604.

2 P. Longo, P. Schmitteckert and K. Busch, Phys. Rev. A, 2011, 83, 063828.

Javier Aizpurua asked: What happens if you insert losses in your description? Have you considered including the losses in your Hamiltonian? What would happen to the ultra-strong coupling if you started putting relevant losses in? Would it then be better to have weaker coupling with weak losses, or to have a modified ultra-strong coupling affected by the strong losses?

David Zueco answered: We did indeed include losses. We considered that the quantum emitter was radiating to other channels. In other words we considered the case of $\beta<1$. The way we did it (beyond the master equation formalism) is described in the Supplementary Material of our recent article. ${ }^{1}$ Essentially, our results are robust against reasonable losses, always such that the coupling emitter-waveguide is stronger than the other decays. It would be interesting to increase the losses, such that they are comparable to the coupling (while the latter is in the ultrastrong regime). We are currently investigating this.

1 E. Sánchez-Burillo, D. Zueco, J. J. García-Ripoll and L. Martin-Moreno, Phys. Rev. Lett., 2014, 113, 263604.

Pablo de Roque commented: Some years ago, J. Shapiro from MIT published a paper $^{1}$ entitled 'Single-photon Kerr nonlinearities do not help quantum 
computation'. In that paper the author gives a theoretical description of photonphoton interaction mediated by atomic nonlinearities. This theoretical description is within the framework of multi-mode treatment of single-photon nonlinearities, as opposed to common single-mode descriptions. He concludes that when taking into account the interaction of non-instantaneous nonlinearities with photon wave packets, the fidelities of the non-linear gates are not sufficient to implement any scalable quantum architecture, since the mentioned fidelities are not above the limits for applying quantum error correction protocols. My question is whether your formalism takes into account those finite-time responses. Conversely, I would like to have your opinion on whether the architecture proposed in your paper (Faraday Discuss., 2015, DOI: 10.1039/C4FD00206G) would be satisfactory for a scalable quantum computer.

1 J. H. Shapiro, Phys. Rev. A, 2006, 73, 062305.

David Zueco responded: Unfortunately I am not an expert on quantum information processing. Thus, I do not know how to answer your question. If we consider finite-time responses, I would like to emphasize that we solve "exactly" the interaction of a waveguide with a quantum emitter. In our numerical simulations we do not make any Markovian or weak coupling approximations.

Pablo de Roque remarked: I would like to have your opinion on the feasibility of having g-factors as high as the ones normally present in theoretical predictions with atoms at optical frequencies, since coupling strength is proportional to the dipole moment, and those quantities are an order of magnitude smaller for atoms in the optical regime than for artificial superconducting qubits.

David Zueco replied: As you said, with artificial atoms (e.g. superconducting circuits) very high coupling strengths are possible. I recently heard that experimentalists are reaching $60 \%$ coupling (compared to the level splitting of the atom). In "natural" emitters, I do not know. I am just involved in some experimental collaboration with magnetic molecules coupled to superconducting cavities. They are trying to reach strong coupling (5-10\% coupling) between the cavity and hundreds of molecules. Never with a single molecule.

F. Javier García de Abajo commented: Can dissipation play an important role in the system that you are studying? Can you use a traditional approach to include dissipation, such as density matrix or Monte Carlo? Also, how valid is the approximation of having Markovian processes in the ultrastrong coupling limit that you are considering? How can dissipation to a bath be realistically described?

David Zueco answered: In a previous paper,${ }^{1}$ we have considered dissipation. We studied the case where the qubit dissipates to other channels. Our calculations considered Ohmic spectral density and that the losses were smaller than the coupling to the line. Markovianity is a property of the bath. It is also related to a weak (system-bath) coupling limit, the so-called Davies limit. In principle, the Markovian character is independent of the system itself. Therefore, I do not see any point of considering if the system (qubit + cavity) is in the ultrastrong regime or not. Obviously the spectrum of the system (being in the ultra or not) will change 
and this could change the range of parameters where the system is weakly coupled to the bath. In other words, ultrastrong coupling is a property of the system. Whether this ultrastrong coupled system is coupled in a Markovian or non-Markovian way is another story. It is the way how it is coupled to the bath. If your question is about a dissipative theory of a system beyond weak (system-bath) coupling the answer is that it depends. In the literature, work already exists on this limit, by working within the path integral formalism for dissipative systems.

1 E. Sánchez-Burillo, D. Zueco, J. J. García-Ripoll and L. Martin-Moreno, Phys. Rev. Lett., 2014, 113, 263604.

Vladimir Bochenkov opened a general discussion of the paper by Alessandro Belardini: What is the nature of the optical activity in your system?

Alessandro Belardini answered: Our samples can be viewed as metasurfaces, so we cannot consider a propagation of the electromagnetic field through effective optically active media. Nevertheless a circular dichroism has been measured, thus indicating a geometrical extrinsic chiral response. This property originates from the relative orientation of a triad of vectors describing the direction of the wires, the direction of the impinging light and the direction of the surface normal. ${ }^{1}$

1 E. Plum, X.-X. Liu, V. A. Fedotov, Y. Chen, D. P. Tsai and N. I. Zheludev, Phys. Rev. Lett., 2009, 102, 113902.

Felix Benz commented: How well-defined is the tilt angle of the nanowires and how sensitive is the measurement to slight local variations? Is the homogeneity influenced by the growth temperature?

Alessandro Belardini replied: From analysing the SEM images of our samples, the tilt angle appears to be $60^{\circ}$ with respect to the surface normal, with a symmetric distribution of approximately $10^{\circ}$. Measurements performed in different portions of the samples do not show substantial differences, revealing the good homogeneity of the samples with respect to the pump spot size $(0.5 \mathrm{~mm}$ diameter). Unfortunately, we do not have samples with different tilt angles in order to investigate the behaviour as a function of the tilt angle. The substrate temperature does not affect the average wires orientation, but the spread of the angular distribution as shown by SEM images.

Niek van Hulst commented: The generated second harmonic will depend on plasmonic resonances with either fundamental or second harmonic wavelength. What is the typical length of the nanowires? Do you see any resonance effect as a function of the length?

Alessandro Belardini answered: From SEM images, the typical length of the wires is $400 \mathrm{~nm}$ for both samples. The linear transmission spectra do not display relevant resonant behaviour neither at $800 \mathrm{~nm}$ (pump) nor at $400 \mathrm{~nm}$ (SHG signal); this is also confirmed by the SHG signal intensity that results being only 2.5 times the signal generated by a flat Au layer. 
Niek van Hulst remarked: The nanowires are made of bulk polycrystalline gold, with a zero $\chi^{2}$ susceptibility. Thus what is the origin of the second harmonic? A surface effect, roughness or potentially a bulk component?

Alessandro Belardini answered: Due to the high surface $v s$. volume ratio we are pretty sure that the main contribution to our signal is related to the surface nonlinear response. We can expect a weak contribution from the magnetic dipole non-linear response from the bulk. Anyway it is not possible to isolate bulk nonlinear contributions in our experimental setup.

Viktor Podolskiy asked: It would be interesting to see the comparison of linear properties of your tilted wires to the predictions of effective medium theories (EMTs). Earlier EMTs have been developed by Mario Silveirinha; he has considered mostly high-conductivity wires, with emphasis on the low frequency regime. Interesting effects, such as inverted rainbows, have been predicted for tilted crossed wire metamaterials; ${ }^{1}$ I'm not sure these will survive for tilted wires that are not crossed. More recently, our group has developed a nonlocal EMT with emphasis on the high-frequency, plasmonic response. ${ }^{2}$

1 M. G. Silveirinha, Phys. Rev. Lett., 2009, 102, 193903.

2 B. M. Wells, A. V. Zayats and V. A. Podolskiy, Phys. Rev. B, 2014, 89, 035111.

Alessandro Belardini responded: We are completing the linear spectral characterisation, and then we will investigate the applicability of the EMT to our samples. It will be interesting to extend your results to our tilted nanowires.

Olivier Martin commented: SHG strongly depends on where you look at it; am I correct in understanding that you always measure SHG from the side of your sample? Have you ever considered looking at different angles and wouldn't this provide additional information on your sample, including on the orientation and homogeneity of the nanowires, since they can influence the SHG (see e.g. "Ultrasensitive optical shape characterization of gold nanoantennas using second harmonic generation"1).

1 J. Butet, K. Thyagarajan and O. J. F. Martin, Nano Lett., 2013, 13, 1787.

Alessandro Belardini responded: We measured SHG at the reflection angle for different incidence angles (ranging from $15^{\circ}$ to $65^{\circ}$ ), but we did not check the SHG out of the reflection angle: this is an interesting suggestion.

Samir Kumar communicated: The authors have grown Au nanowires at two different temperatures ( $100 \mathrm{~K}$ and $300 \mathrm{~K}$ ) by oblique angle deposition (OAD). From SEM images it seems that the nanowires grown at $300 \mathrm{~K}$ are more sharp and well separated. The effect of substrate temperature on the nanostructures grown by OAD has been studied very well by various groups. It is well known that at low substrate temperature the nanorods are sharper and longer than the nanorods grown at higher temperatures because of the limited surface diffusion. But your nanowires are the opposite of what has been observed. I want to know why your structures are contradicting the already established studies? 
Alessandro Belardini communicated in reply: At first sight, we were surprised by our results as well. However, carefully analysing the SEM images, we noticed that the $100 \mathrm{~K}$ sample presents thinner wire diameters $(10-20 \mathrm{~nm})$ due to the low surface diffusion. Then, as the wires length increases during the growth process, groups of wires collapse forming pyramidal arrangements (bunches) of wires with a thicker base of $200 \mathrm{~nm}$ diameter (collecting more isolated wires) and with a tip of $80 \mathrm{~nm}$ diameter where the wires are connected. On the other hand, in the $300 \mathrm{~K}$ sample, the wires grow as larger columns from the base $(30-40 \mathrm{~nm})$ and are stable along their length (approx. $400 \mathrm{~nm}$ ), thus remaining isolated wires.

Niek van Hulst opened a general discussion of the paper by Michael Flatté: You mention that YIG particles at the liquid-liquid interface cause the Faraday effect. Do these particles have to be oriented for the macroscopic effect to occur? Do you need an external field for orientation?

Michael Flatté replied: The particles themselves do not need to be oriented, but the magnetization of the particles does need to be oriented. This can be done with an external magnetic field. YIG has a very low coercive field, especially for near-spherical particles, so the magnetization can be oriented either parallel to the interface or perpendicular to it with a modest magnetic field.

Bart de Nijs asked: You show in your article (Faraday Discuss., 2015, DOI: 10.1039/C4FD00210E) and presentation a very nice method of controlling the polarisation of light using nanoparticles attaching and detaching from a liquidliquid interface. But you also show that this attachment then occurs at a certain potential. Does the detachment happen at the same potential or will there be some form of hysteresis? How reversible would the system be?

Michael Flatté replied: As we have found in previous studies (e.g. Fig. 2 of ref. 5 in our paper, Faraday Discuss., 2015, DOI: 10.1039/C4FD00210E), it is possible at intermediate voltages to create a positive potential at the interface, but separated from the bulk by a large potential barrier. This situation can produce large hysteresis. Thus it would be most favorable for switching behavior to avoid the use of intermediate voltages for switching.

Bart de Nijs said: Is there any idea with what speed this switching can be done?

Michael Flatté answered: Yes, in previous studies (ref. 5 in our paper, Faraday Discuss., 2015, DOI: 10.1039/C4FD00210E) we have estimated the speed with which a single layer of nanoparticles of similar size can be brought to, or away from an interface. Depending on system parameters, this time lies roughly between 0.1-1 s. However, using voltage pulsing techniques or stirring this can be sped up. For more details see ref. 1 and 2 below.

1 A. A. Kornyshev, A. R. Kucernak, M. Marinescu, C. W. Monroe, A. E. S. Sleightholme and M. Urbakh, J. Phys. Chem. C, 2010, 114, 14885.

2 M. Marinescu, M. Urbakh, T. Barnea, A. R. Kucernak and A. A. Kornyshev, J. Phys. Chem. C, 2010, 114, 22558. 
Alexei A Kornyshev commented: Nanoparticles localise spontaneously in arrays at liquid interfaces, if the charge of their ligands and the concentration of electrolyte which screens the electrostatic repulsion but is not too strong to allow their fusion in the bulk are properly adjusted. ${ }^{1}$ To move such particles from the interface, applying voltage across it, is a tricky task and has been achieved so far only for small particles, ${ }^{2}$ because the capillary forces that keep them at the interface are very large. One of the ways to reach electrical control over the population of nanoparticles at the interface will be to start with such conditions of the electrolyte concentration and $\mathrm{pH}$ for which the nanoparticles do not yet selfassemble at the interface, but are very close to that situation. Since the electric field across the interface is localized within the back-to-back electrical double layers, the particles will reach the surface via random diffusion but not migration, and will be trapped at the interface by the electric field localized there. All this was discussed in detail in our feature article in the Journal of Physical Chemistry C. ${ }^{3}$

1 M. P. Cecchini, V. A. Turek, J. Paget, A. A. Kornyshev and J. B. Edel, Nat. Mater., 2013, 12, 165.

2 J. B. Edel, A. A. Kornyshev and M. Urbakh, ACS Nano, 2013, 7, 9526.

3 M. E. Flatté, A. A. Kornyshev and M. Urbakh, J. Phys. Chem. C, 2010, 114, 1735.

Thomas Ebbesen asked: Can you clarify the size of the setup? Would it work faster if the dimensions were smaller?

Michael Flatté replied: The size of the setup in the lateral direction for light impinging perpendicular to the interface would be the spot size of the light beam, so a minimum size would be a few square microns. For the cell in the vertical direction the minimum thickness required is that to dilute the nanoparticles into the bulk, and bring them back to the interface, within $\sim 0.1-1 \mathrm{~s}$. That thickness is about 2 microns (ref. 5 in our paper, Faraday Discuss., 2015, DOI: 10.1039/C4FD00210E). Similar sizes are appropriate for the waveguide geometry also presented.

Thomas Ebbesen remarked: What's the size of your particles and the concentration of the electrolyte? The electrolyte can of course screen the field enormously. Can you work with lower electrolyte concentrations?

Michael Flatté answered: Yes, the size of the particles cannot be too large or they will become trapped at the interface and cannot be removed, presumably not larger than $20-40 \mathrm{~nm}$ in diameter; however, manipulation with liquid-liquid interface tension (reducing it) may relax this constraint. The electrolyte concentration cannot be too high or the electric field becomes localized to the interface alone. Although the electric field gets larger by making the double layer at the liquid-liquid interface more compact, it will act at a smaller number of charges on the nanoparticles, and the effect of the field may eventually get weaker. We assume a concentration of $5 \times 10^{-3} \mathrm{M}$, the same as in ref. 22 in our paper (Faraday Discuss., 2015, DOI: 10.1039/C4FD00210E).

Alexei A Kornyshev added: Experiments with nanoparticles that can provide Faraday rotation have not yet been performed. But for metallic particles that we used to prove SERS at the liquid-liquid interface, the sizes were $18 \mathrm{~nm}$ in diameter, with presumably about $2 \mathrm{~nm}$ of surface-to-surface separation in the 
settled array, which warranted detection of analytes down to femtomolar amounts in $1 \mathrm{~cm}^{3}$. This was yet not an electrochemical cell, so there was no controllable electric field across the interface. The formation of the array was facilitated by mild centrifugation or just shaking, and took about $1 \mathrm{s.}{ }^{1}$

1 M. P. Cecchini, V. A. Turek, J. Paget, A. A. Kornyshev and J. B. Edel, Nat. Mater., 2013, 12, 165.

Andrew Mount asked: Is the radius, $R$, of the nanoparticle a hydrated radius, and is this likely to include e.g. the Stern layer of ions? Is the effective radius of the particle also likely to change with the ionic strength (controlled by the concentration of background electrolyte)? This one might expect as the optimum spacing between particles at monolayer coverage (their effective size) should increase with an increase in electrostatic repulsion between neighbouring nanoparticles; this could be caused by an increasing surface charge, which could be controlled by the contribution from the number of strongly electrostatically bound or adsorbed surface ions from the electrolyte, which could vary with electrolyte concentration. If so, what effect would this have on the calculated properties of the system, and to what extent has this been accounted for by $e . g$. incorporating the pairwise interaction of localised nanoparticles via screened electrostatic forces?

Michael Flatte responded: The radius of the nanoparticles is the radius of the YIG material. The layer of ligands around the YIG is anticipated to be very thin compared with the nanoparticle radius, as would any hydrated layer. Although the nanoparticle radius would not change due to a change in the ionic strength, there would be a change in the maximum allowed coverage of the interface. This has recently been demonstrated experimentally for gold nanoparticles. ${ }^{1}$

1 V. A. Turek, M. P. Cecchini, J. Paget, A. R. Kucernak, A. A. Kornyshev and J. B. Edel, ACS Nano, 2012, 9, 7789.

Mikhail Noginov commented: Why do the nanoparticles assembled at the surface behave differently from the nanoparticles in the volume? Also, what is the surface filling factor for the nanoparticles?

Michael Flatté answered: For magnetization perpendicular to the interface, they behave differently than in the bulk because each nanoparticle also responds to the polarization field from the neighboring nanoparticles. This additional contribution from the polarization fields produces a larger dielectric response which yields a larger Faraday rotation. For magnetization parallel to the interface, these polarization fields produce a large birefringence that quenches the Faraday rotation. The surface filling factor is given by the coverage, $\Gamma$.

James T. Hugall opened a general discussion of the paper by David Richards: Using the lifetime imaging technique you describe, at any given lateral position in a sample, as soon as there are multiple fluorophores at multiple heights (axial positions), then the lifetime recorded will be an average over the many different fluorophores, and any precise height information/resolution will be lost. Therefore, this technique seems suited only to systems with a very well-defined, and singular, functionalised $2 \mathrm{D}$ surface within a $3 \mathrm{D}$ structure. In any real system, this 
requires that the fluorophores are highly localised to a $2 \mathrm{D}$ surface and thus requires prior knowledge of the system and a system in which there is no existence of similarly functionalised surfaces at different heights. Could you comment on this apparent limitation and the validity of this process for real biological systems? Are there ways to overcome it?

David Richards responded: Indeed, if fluorophores are located over a range of distances from the metal surface, the lifetime recorded will be an average resulting from the integrated fluorescence signal from the distribution of fluorophores. However, for studies of the cellular plasma membrane, the fluorescence lifetime-encoded axial position information provided by the technique can provide a valuable probe of the cellular plasma membrane. We have provided one example in this paper (Faraday Discuss., 2015, DOI: 10.1039/C4FD00198B), in which we are able to map, through detection of the receptor CXCR4, the height of the plasma membrane above a nanostructured silver substrate. Elsewhere, we have demonstrated how this approach is able to provide a sensitive assay of receptor-mediated endocytosis, ${ }^{1,2}$ both for nanostructured and flat metal films. For the nanostructured silver films the fluorescence intensity is also enhanced at the surface relative to distances $>100 \mathrm{~nm}$, commensurate with the lifetime modification, ${ }^{2}$ providing an additional weighting of the lifetime to those fluorophores closest to the surface.

1 N. I. Cade, G. Fruhwirth, S. J. Archibald, T. Ng and D. Richards, Biophys. J., 2010, 98, 2752. 2 N. I. Cade, G. Fruhwirth, T. Ng and D. Richards, J. Phys. Chem. Lett., 2013, 4, 3402.

Niek van Hulst remarked: Lifetime is a very powerful observable as it depends on an enormous amount of parameters, e.g. it matters whether a molecule is parallel or perpendicular to the surface, differences in chemical environment have an effect, quenching to metal reduces the lifetime, and local density of states effects enhance or shorten the lifetime. Thus many parameters are influential and several assumptions are needed to use lifetime as an indicator of distance to a metal surface. Next, what is the range as a distance sensor? Quenching will dominate at the lowest few nanometers, yet at larger distance LDOS effects will take over. Thus surely you do observe distance-dependent effects, however how can this be made quantitative?

David Richards responded: We will first remark that measurements of fluorescence intensity, used to encode distance in TIRF microscopy, for example, are affected very significantly by variations in fluorophore and photobleaching, to both of which lifetime is relatively insensitive. Of those parameters which do affect lifetime, we are exploiting the distance-dependent changes in radiative and non-radiative lifetime, through local density of states effects and quenching, as a tool to specifically encode distance. From calculations of the distance-dependence of fluorescence lifetime, which provide strong agreement with our experimental observations, we have identified that in the case of the nanostructured silver substrate we present, enhancement of radiative decay rate dominates over the relevant distance range. ${ }^{1}$ As we show in the present paper (Faraday Discuss., 2015, DOI: $10.1039 / \mathrm{C} 4 \mathrm{FD} 00198 \mathrm{~B})$, the distance-dependence can be quantified using a calibration sample. Lifetime is indeed dependent on molecular 
orientation, but in our measurements we expect both a significant population of fluorophores within the focal area of excitation, and rapid fluorophore rotation, such that we are sensitive only to an average over all fluorophore orientations.

1 N. I. Cade, G. Fruhwirth, T. Ng and D. Richards, J. Phys. Chem. Lett., 2013, 4, 3402.

Niek van Hulst asked: The lifetime changes are particularly strong over the first few nanometers. I assume, looking at cellular dynamics, you will mainly observe intracellular membrane dynamics close to the metallic substrate, which could be useful for the study of membrane organisation?

David Richards responded: From the calibration measurements we have performed we find that the lifetime changes are particularly strong over the first 100 $\mathrm{nm}$ away from the plasmonic surface. In particular, we have demonstrated elsewhere that this technique provides sensitive detection of the internalisation of fluorescently labelled membrane receptors, providing a sensitive assay of receptor-mediated endocytosis. ${ }^{1,2}$

1 N. I. Cade, G. Fruhwirth, S. J. Archibald, T. Ng and D. Richards, Biophys. J., 2010, 98, 2752. 2 N. I. Cade, G. Fruhwirth, T. Ng and D. Richards, J. Phys. Chem. Lett., 2013, 4, 3402.

Martin Moskovits asked: This is a clever experiment in which you were able to obtain what is in essence tomographic structural information at the nanometer scale, by making use of the fact that the fluorescence lifetime decreases markedly as the fluorescence source approaches the cell-metal interface. This phenomenon should also occur for a flat metal surface rather than a nanostructured metal surface, whose geometry introduces both preparative complexities and uncertainty in position along the normal direction. Why is it necessary or beneficial to use a nanostructured surface for such experiments?

David Richards responded: We do indeed also demonstrate in our paper (Faraday Discuss., 2015, DOI: 10.1039/C4FD00198B) the measurement of axial position from a flat gold surface with super-resolution sensitivity. However, because fluorescence lifetime is reduced only through an enhancement in nonradiative decay, leading to fluorescence quenching, we find that the resultant reduction in signal makes the determination of distance unreliable for separations very close to the metal surface. In contrast, a nanostructured silver film leads to an enhancement in fluorescence signal close to the surface, with fluorescence lifetime thereby providing a reliable measure of axial position with a sensitivity down to $6 \mathrm{~nm}$. A thin film nanostructure also provides the additional advantage of being able to perform measurements on cells in a conventional inverted microscope geometry, whereas flat metal surfaces with poor optical transmission require illumination and detection from above the sample.

Martin Moskovits said: Benefitting from the fluorescence enhancement is helpful, but that would surely also restrict your observational territory to the lower portion of the cell. A great deal of biology occurs in the inaccessible portion of the cell.

David Richards replied: Because the technique we present uses conventional confocal microscopy, we are able to probe the whole body of the cell, with the 
measurement of fluorescence lifetime providing super-resolution distance discrimination within the first $100 \mathrm{~nm}$. For example, as we demonstrate, this enables a straightforward assay of endocytosis, providing discrimination between e-GFP labelled proteins in the cell membrane and those that have been internalised. We note that, in contrast, in the case of total internal reflection fluorescence microscopy (TIRFM), widely used in studies of the cellular membrane, the observational territory is indeed restricted to the lower portion of the cell.

F. Javier García de Abajo enquired: Could you speculate on how much the combination of lateral and radial resolutions can be improved? The former could go down to $\sim 10 \mathrm{~nm}$ by collecting many images made with one or a few emitting fluorophores each (i.e., like in the stochastic optical reconstruction microscopy (STORM) technique), whereas the latter could rely on the impressive determination of the lifetime in your technique. Could these two perhaps be combined by going down to one or a few emitters per frame and looking not only at the lateral intensity profile for each individual emitter but also at how many photons are emanating from it, which should be connected with its lifetime, and this in turn with the presence of plasmonic decay channels (i.e., its distance to the metal substrate)?

David Richards replied: We have estimated that the technique we present provides $6 \mathrm{~nm}$ axial distance sensitivity over a range of $100 \mathrm{~nm}$ from the surface (i.e. radial distance sensitivity, in the case of an isolated silver nanoparticle). This translates to precision for the determination of the height of a sheet of fluorophores, such as for the measurement of a cellular membrane presented in this paper (although the lateral resolution of the technique is still diffraction limited to $\sim 200 \mathrm{~nm}$ ). In this case the axial sensitivity is limited by the distance-dependence characteristics of the fluorescence lifetime and its determination; hence it could be improved further with tuning of the plasmonic substrate, reducing the instrumental response time, and using improved fluorescence decay fitting algorithms for low photon numbers (e.g. a Bayesian approach). On the other hand, the technique we present is not able to provide such axial resolution within a $3 \mathrm{D}$ volume of fluorophores, as is the case with localisation-based super-resolution microscopies, such as STORM and photoactivation localisation microscopy (PALM). However, as it is based around well-established fluorescence lifetime imaging (FLIM), it is able to offer acquisition times which are compatible with live cell imaging, generally not possible with PALM and STORM because of the need to measure large numbers of single-molecule images using those methods. To bring the two approaches together, the detection of single fluorophores presents first a challenge in the measurement of fluorescence lifetime decays using time-correlated single photon-counting, in particular within a full-frame wide-field modality required by PALM and STORM. Moreover, we would contend that the 3D resolution provided by these techniques would be superior to the plasmon-assisted technique we present, while the advantage of compatibility with conventional confocal microscopy in live cell imaging would be lost.

Niek van Hulst commented: As to combining your lifetime method with localisation microscopy, I think you should not be so pessimistic. Typically about $\sim 1000$ photocounts are enough for a single molecule to determine lifetime, or 
changes in lifetime. Thus, with regard to photon budget, one has sufficient signal to do both lifetime and STORM; the issue is more in single-point confocal versus wide-field microscopy. I do see an issue in using localisation microscopy with plasmonic structures to measure the position of emitters. The emission will couple to the plasmonic mode, thus one will localise the plasmonic mode instead of the molecule. A shift of many nanometers can occur due to the metal, and thus usually STORM on plasmonic-enhanced systems does go wrong due to the coupling to the plasmonic mode.

Olivier Martin addressed David Richards: The new axial tomography imaging technique you have established relies on the plasmonic substrate which serves as a reference for the signal measured for the cell that rests on that substrate, and the information recorded depends on the distance from that substrate. Recent experiments have used plasmonic nanoparticles to label the membrane of a cell; ${ }^{1}$ couldn't one combine this labelling technique with your approach, to provide additional plasmonic references throughout the cell?

1 J. Wang, S. V. Boriskina, H. Wang and B. M. Reinhard, ACS Nano, 2011, 5, 6619.

David Richards answered: With the approach discussed in the present paper (Faraday Discuss., 2015, DOI: 10.1039/C4FD00198B) we assume there is a uniform plasmonic substrate over the whole sample (confirmed by atomic force microscopy and absorption spectroscopy), and hence do not need to consider any additional affinity factors due to introducing labelled nanoparticles that target specific sites. Nevertheless, we note that for the very high nanoparticle labelling densities reported by Wang et al., a plasmonic sheet would be effectively created over the apical membrane; this would provide a means to discriminate fluorophores (through their lifetime) in close proximity to the membrane from those deeper within the body of the cell, in a similar manner to that which we have presented for the basal cell membrane, with application in, e.g., assays of receptor-mediated endocytosis. ${ }^{1}$

1 N. I. Cade, G. Fruhwirth, S. J. Archibald, T. Ng and D. Richards, Biophys. J., 2010, 98, 2752.

Jeremy Baumberg asked: On the physics side there are strengths and weaknesses of using silver grains. But biologically the cells react very differently to smooth and rough surfaces, would you like to comment on that? Particularly thinking about roughness, length scales...

David Richards replied: We would first highlight that usually silver is considered to be toxic to cells. However, we have applied a protocol, identified in the present paper (Faraday Discuss., 2015, DOI: 10.1039/C4FD00198B), whereby a coating of $\mathrm{N}$-(2-mercaptopropionyl)glycine (MPG) over the silver plasmonic substrate permits normal cell growth. Due to its free -SH groups MPG binds well to silver, thereby inhibiting the toxic effect of silver. We do not have information about whether or how the MPG modifies the nanoscale morphology of the surface, but note that we did not observe qualitatively any obvious growth inhibition or change of the morphology of MTLn3E adenocarcinoma cells cultured on these surfaces. 
Jeremy Baumberg responded: But particularly the conformation of a cell at the bottom which you can't normally measure, how does it adhere to the surface, and may it respond differently to different materials and structures?

David Richards replied: This could indeed be investigated. In principle other surface functionalisation techniques could be used to provide a thin barrier layer between the silver film and cellular membrane (e.g. fibronectin, PEGylation) without adversely affecting the plasmonic response. However, care is required to ensure there is no leakage of silver, prevented through the use of MPG as a barrier.

Katarzyna Matczyszyn opened a general discussion of the paper by Yu Chen: What are the dimensions of your nanorods? Do you play with various sizes or coatings? Also, how do you do the surface chemistry? For the functionality of the probes, the length of the DNA matters, were you dealing with the specific sequencing?

Yu Chen responded: The rods are about $40 \mathrm{~nm}$ long and $12 \mathrm{~nm}$ in diameter. We have studied rods of different aspect ratios and coatings (for details, please see ref. 7 in our paper, Faraday Discuss., 2015, DOI: 10.1039/C4FD00199K). In this work, the hairpin was designed to target c-myc, with a loop containing 17-21 bases.

Katarzyna Matczyszyn said: How do you control the distance between the nanorods, though it depends on the sequence and persistence length of the DNA? Do you control the number of RNA molecules attached to a single nanorod?

Yu Chen answered: The nanorods are well-separated in the solution of low concentration, and the number of hairpins attached to a single nanorod can be tuned by changing the ratio of nanorods to hairpins in the salting aging process.

Olivier Martin asked: Endocytosis is a very complex process that depends on many conditions, including the charges on the particle, the $\mathrm{pH}$, the hydrodynamic radius, etc. Do you think that the technique you have developed could improve understanding of the role of these different parameters and the monitoring of endocytosis as it happens? Also, is it likely that the energy transfer between the gold nanorods and the fluorophores is influenced by some of these parameters that influence endocytosis (charge, $\mathrm{pH}$, etc.), which could then interfere with this type of measurements?

Yu Chen replied: Gold nanorods can be synthesized with different sizes/aspect ratios, and further functionalized with different surface chemistry/charge states, so it is possible to apply this method in living cells and study the factors that influence the endocytosis process. Fluorescence lifetime can be sensitive to local environment, e.g., viscosity, $\mathrm{pH}$ etc., in addition to energy transfer. Therefore, one should be careful about choosing a fluorophore, e.g., using a fluorophore that is less sensitive to $\mathrm{pH}$.

Jeremy Baumberg commented: Do we even know that the gold nanoparticles go in the same places as the gold nanorods? You're assuming you're tracking the 
same sequence of events-do we know that yet? Does the aspect ratio have some effect?

Yu Chen replied: Endocytosis is an important pathway for the uptake of gold nanoparticles, including gold nanorods. This work shows a potential way to study the intracellular pathway of gold nanorods and this method can also be applied to other internalisation processes. Regarding the effect of the aspect ratio, previous studies on the internalisation of gold nanorods with aspect ratios from 2 to 5 didn't find any obvious differences.

Mikhail Noginov asked: How do surface plasmons affect the energy transfer? What is the mechanism for this?

Yu Chen replied: Surface plasmons enhance the excitation of the molecules and also modify the non-radiative decay rate.

Hasan Aldewachi said: Have you studied the effects of different pathological conditions on the endocytosis process of the nanorod-dye combination, as these conditions might affect the mechanism by which endocytosis happens? Also, you mentioned that this combination is less toxic towards certain cells, does that mean that this combination is not applicable for all cell types for imaging and sensing?

Yu Chen responded: We haven't studied the effect of pathological conditions on the endocytosis process. The method discussed in this paper can be applied to this kind of study. The cytotoxicity of gold nanorods depends on the dose and on the surface coating (please see ref. 7 in our paper, Faraday Discuss., 2015, DOI: 10.1039/C4FD00199K). So we can use this method in cells that are less tolerant to CTAB-capped gold nanorods, as shown in this paper.

Hasan Aldewachi commented: Why do you prefer to use the nanorods instead of nanoparticle spheres? The latter don't need to be prepared in CTAB, could it be more cell-friendly?

Yu Chen replied: Citrate-stabilized nanospheres are more cell-friendly than CTAB-gold nanorods are. However, gold nanorods have many advantages, including a tunable longitudinal surface plasmon mode and strong two-photon luminescence, etc.

Jeremy Baumberg asked: When you're looking at the hairpin system, the one thing you're going to be very sensitive to is heating of the system. Do you see any effects from that? Do you get enough photons out when the power is low enough to avoid heating?

Yu Chen responded: Thermal stability of the hairpin is an important issue and one of the factors to be considered in hairpin design. This paper (Faraday Discuss., 2015, DOI: 10.1039/C4FD00199K) shows three stable structures and one less stable one. With an appropriate design, reliable signals can be obtained under normal measurement conditions. 
Mikhail Noginov remarked: In equation 1 as presented in your slides $\left(k_{T}(r)=\frac{1}{\tau_{D}}\left(\frac{R_{0}}{r}\right)^{6}\right), k$ has units of $\mathrm{s}^{-1}$. In equation $2\left(k_{T}=1-\frac{F_{D A}}{F_{D}}=1-\frac{\tau_{D A}}{\tau_{D}}\right), k$ is dimensionless. Two different quantities should not be denoted by the same letter.

Yu Chen replied: The second one should be $\varepsilon\left(\varepsilon=1-\frac{F_{D A}}{F_{D}}=1-\frac{\tau_{D A}}{\tau_{D}}\right)$.

Olivier Martin commented: The imaging techniques presented in the papers from David Richards and Yu Chen (Faraday Discuss., 2015, DOI: 10.1039/ C4FD00198B and Faraday Discuss., 2015, DOI: 10.1039/C4FD00199K) have great potential for uncovering some of the complex mechanistic processes taking place in living cells; in particular to shed light on the dynamics of these processes. Do you think that both techniques can be applied to living cells? If so, how fast can they perform? What kind of typical acquisition time can be reached, maybe by compromising on the spatial resolution?

Yu Chen replied: This technique can be applied in living cells. The typical acquisition time for a good quality image is a few minutes, though it can be reduced to half a minute by compromising on the spatial resolution. FLIM acquisition at $500 \mathrm{~ms}$ has recently been reported, by parallelizing the excitation and detection processes. ${ }^{1}$

1 S. P. Poland et al., Biomed. Opt. Express, 2015, 6, 277.

David Richards answered: FLIM imaging of living cells is relatively easily achievable at approximately video rates (see ref. 1 and other more recent papers by French and co-workers), with the acquisition time ultimately limited by the available photon budget. This results in a compromise between speed, the accuracy of determination of the fluorescence lifetime and the lateral image resolution, and the usual imaging restrictions such as photo-bleaching.

1 D. M. Grant et al., Optics Express, 2007, 15, 15656.

James Hutchison asked: I see you require three exponential components to fit your decays, and attribute them to energy transfer of various efficiencies or to scattering from the gold core. How confident are you in attributing each component of these decays to specific processes, given that (I assume) you float 6 variables to achieve a fit?

Yu Chen answered: Each rod has multiple dyes in different orientations and at different locations on the gold surface. Multiple exponential decays would be expected. The two lifetime components in the ns and sub ns range can be attributed to Cy5. The first lifetime component in the case of the less stable hairpin structure is close to the lifetime of free Cy5. Therefore the reduction in lifetime indicates the energy transfer and is used for comparison. The short lifetime component in the tens of ps region is likely due to scattering from gold cores.

Prineha Narang was invited to make some remarks to open a general discussion of the paper by Martin Moskovits, summarised as follows: To put these 
remarks into context, the decay of surface plasmon resonances is usually a detriment in the field of plasmonics, but the possibility to capture the energy normally lost to heat would open new opportunities in photon sensors, energy conversion devices and switching. In the context of hot-electron devices, the large extinction cross-section at a surface plasmon resonance enables nanostructures to absorb a significant fraction of the solar spectrum in very thin films. Despite the significant experimental work in this direction, a complete theoretical understanding of plasmon-driven hot carrier generation with electronic structure details has been elusive. Theoretical studies of plasmonic systems have traditionally focused on their optical response, including jellium models of nanostructured systems. While extremely valuable, these models do not capture the material dependence of this process and miss interband transitions in noble metals. ${ }^{1}$ Recently we analyzed the quantum decay of surface plasmon polaritons and found that the prompt distribution of generated carriers is extremely sensitive to the energy band structure of the plasmonic material. ${ }^{2}$ In this context, we view processes involving plasmons, electrons and phonons within a Feynman diagrammatic approach and treat plasmonic hot carrier generation fully from first principles. Built upon this general theoretical and computational framework, we will present results for $\mathrm{Cu}, \mathrm{Ag}$ and $\mathrm{Au}$ on higher order processes such as multiplasmon decays in metals that are critical for plasmon-driven upconversion and non-linear plasmonics. ${ }^{3}$ The role of phonon-assisted intraband contributions in plasmonic hot carrier generation calculated from $a b$ initio calculations will be shown. I discussed the implications of multi-plasmon decays on the design of hot carrier devices and plasmon-driven chemistry applications.

1 A. J. Leenheer, P. Narang, N. S. Lewis and H. A. Atwater, J. Appl. Phys., 2014, 115, 134301.

2 R. Sundararaman, P. Narang, A. S. Jermyn, W. A. Goddard III and H. A. Atwater, Nat. Commun., 2014, 5, 5788.

3 P. Narang, R. Sundararaman, A. S. Jermyn, A. M. Brown, W. A. Goddard III and H. A. Atwater, in preparation.

Alexei A Kornyshev opened a general discussion of the paper by Martin Moskovits $\dagger$ : In the effects that you have described, is there anything principally different from the photoemission from metal into electrolyte? This subject was very popular in the 1970s, due to the works of Brodsky, Gurevich, Rotenberg and Pleskov in the Soviet Union and Alan Bard in the USA (for a review see Ref. 1), but pioneered perhaps in the less-known work of Geoffrey Barker (the Electrochemistry Medal of the Royal Society of Chemistry is called the "Geoffrey Barker Medal").

1 A. M. Brodsky and Y. V. Pleskov, Prog. Surf. Sci., 1972, 2, 1.

Martin Moskovits answered: As you have correctly pointed out, such hotcarrier internal photoemission, from a metal to a semiconductor or to an electrolyte, has been known for a long time. For example, we have shown in the early 90 's that hot electrons can be extracted out of roughened metal surfaces directly as enhanced and directional photoemission into vacuum. ${ }^{1}$ The directionality was

$\dagger$ The written version of the responses to the questions about Martin Moskovits' paper were completed by Syed Mubeen. 
shown through a supporting theory based on light-induced drift, to indicate that the majority of the hot electrons arrived at the nanoparticle's surface ballistically. To use hot electrons in photovoltaic or photocatalytic systems, however, one needs to fabricate nanostructured devices that present interfaces appropriately chemically modified to extract and use these carriers before they lose their energy as heat. This is due to the short time length scales-approximately tens of nanometers-associated with rapid hot carrier dynamics. Further, when an appropriately nanostructured material (such as silver, gold or palladium as in this case) that can sustain high-quality localized surface plasmon resonances is illuminated with light of an appropriate wavelength, the optical fields radiated by the nanostructure and the incident field can coherently superimpose, redistributing the radiant energy so as to create regions of concentrated electromagnetic energy where the field amplitude can be enhanced by factors of tens or even hundreds, thereby enabling enhanced hot-carrier generation and photoemission. Also, the ability to tune localized surface plasmons in a prescriptive fashion by simply altering the architecture allows one to create panchromatic devices covering the entire solar spectrum.

1 V. M. Shalaev, C. Douketis and M. Moskovits, Phys. Lett. A, 1992, 169, 205.

Thomas Ebbesen commented: I like your idea very much. I've been following a little bit the literature in Japan on this concept but the devices are much more macroscopic. ${ }^{1,2}$ They produce hydrogen and oxygen in the right proportions, and the action spectra follow the surface plasmons resonance even in the IR. It looks ideal; the only problem is the very low quantum yield in their systems. What's the best quantum yield that you have obtained so far and what are the prospects for improving it?

1 Y. Nishijima, K. Ueno, Y. Kotake, K. Murakoshi, H. Inoue and H. Misawa, J. Phys. Chem. Lett., 2012, 3, 1248.

2 Y. Zhong, K. Ueno, Y. Mori, X. Shi, T. Oshikiri, K. Murakoshi, H. Inoue and H. Misawa, Angew. Chem. Int. Ed., 2014, 53, 10350.

Martin Moskovits replied: We have achieved so far a maximum of $1.5 \%$ internal quantum efficiency for solid state photovoltaic devices and $1 \%$ internal quantum efficiency for photocatalytic devices.

Thomas Ebbesen continued: A 1\% quantum yield is already very good. You have the advantage in your system that you store the energy in chemical products. If one converted hydrogen and oxygen using photovoltaics, the overall yields would be much lower. How did you get up to $1 \%$, what did you change?

Martin Moskovits responded: Thank you for your positive comments. Internal quantum efficiency here is the absorbed photon-to-hot-electron injection and collection efficiency of the device, as measured by the currents in the case of PV devices and the amount of chemical products $\left(\mathrm{H}_{2}\right)$ in the case of photocatalytic devices. There are multiple steps that can decrease the quantum efficiency in the case of a plasmon-driven photocatalytic process (some of which can be applied to PV systems as well), such as: (i) the plasmon materials themselves-inefficient coupling of the plasmonic absorber material to the redox couple or to the catalytic 
system; (ii) the hot carriers themselves-inefficiencies in transporting the hot carriers to appropriate electrochemically-active sites to perform desired redox chemistries; (iii) the catalytic systems themselves-inefficiencies of the catalytic systems by themselves; and (iv), the interfacial charge transfer process-inefficiency of the 'electron filter' layer (mostly a semiconductor or insulator) in contact with the plasmonic metal. Consequently, the low efficiencies registered so far for plasmonic devices could be any or all of them. In this study (Faraday Discuss., 2015, DOI: $10.1039 /$ C4FD00185K) we tried to improve the quantum efficiencies by eliminating the intermediate 'electron filter' layer and 'co-catalyst' and used plasmonic metal as a standalone light absorber and catalytic unit. The above results also motivate us to believe that much improvement in efficiencies can be expected if one can better understand the overall chain of events, that begins with photoexcitation of the plasmon and terminates with the production of the desired product (fuel or electricity).

F. Javier García de Abajo commented: You have presented a very inspiring concept. I just have a question about the timescales involved. Let me consider for example a single $2 \mathrm{eV}$ plasmon decaying into an electron-hole pair, with the hole near the Fermi energy, where the density of electron states is large, and the electron is then $2 \mathrm{eV}$ above that level. It takes a few femtoseconds ( $\sim 10 \mathrm{fs}$ in gold) for the electron to release part of its energy, giving rise to a second electron-hole pair. In this cascade process, the number of hot charge carriers is increasing with time, while their average energy goes down, so the sum of their energies is the energy of the original plasmon minus the fraction that is dissipated by coupling to phonons. Although a ballistic regime is maintained during $100 \mathrm{~s}$ of $\mathrm{fs},{ }^{1}$ a diffusive regime is eventually reached. Therefore, we have one electron with $2 \mathrm{eV}$ energy for $\sim 10 \mathrm{fs}$, then two electrons with lower energy for $\sim 10 \mathrm{fs}$, and eventually the energy is dissipated to phonons in a few picoseconds. I am then wondering what the efficiency of the hot electrons is for triggering chemical reactions at the surface of the metal. The penetration of the electron wave function towards the medium outside the metal consists of its evanescent tail, which extends more when the electron has higher energy relative to the Fermi level (i.e., when it is closer to the confining potential barrier). Therefore, I would imagine that hotter electrons are present during the first tens of fs, while much lower-energy electrons are present during the rest of the time required for dissipation into phonons. What is, then, the timescale for efficiently triggering chemical reactions at the particle surface? The above picture could apply to a linear regime (i.e., when the chemical reaction activated by hot electrons is produced at a rate that is linear with the light intensity). It is also possible that for larger light intensities a hot-electron plasma is maintained at a temperature of 100 s of degrees due to the electron-phonon thermal barrier, ${ }^{2}$ so a non-linear rate of chemical reaction is produced. I would appreciate to know your views on these issues.

1 S. D. Brorson, J. G. Fujimoto and E. P. Ippen, Phys. Rev. Lett., 1987, 59, 1962.

2 M. Perner, P. Bost, U. Lemmer, G. von Plessen, J. Feldmann, U. Becker, M. Mennig, M. Schmitt and H. Schmidt, Phys. Rev. Lett., 1997, 78, 2192.

Martin Moskovits answered: Those are very interesting questions. As pointed out, the dephasing times for localized surface plasmon (LSP) resonances are very 
short, in the order of 1-10 femtoseconds, the electron-phonon relaxations are in the order of 1-5 picoseconds and equilibration with its surroundings is achieved at even higher time scales. So the question is, what is the energy distribution of electrons in a plasmonic metal (here Pd) when it is CW illuminated at frequencies corresponding to the LSP of the Pd particle, and what will the energy distribution of the hot electrons be before a great deal of energy has been lost due to electronphonon interactions. To our knowledge, no adequate theoretical treatment has yet been carried out to determine the energy distribution function of the electrons across different time scales-in all fairness though this is a rather formidable time-dependent calculation. However, since the LSP absorption in a plasmonic metal arises from the participation of thousands of virtual single-electron transitions (the transition dipole for the LSP in a single silver nanoparticle is $\sim 20000$ D), we believe that at least one electron (maybe more than one) resulting from plasmon dephasing will occupy an energy level as high as $h \omega_{\text {sp }}$ above the Fermi energy. Moreover, if the Pd particle is irradiated for a period of time (CW illumination), a new plasmon will be continuously excited; this will create hot electrons at rates much higher than electron-phonon relaxation rates, resulting in a steady state with many hot electrons occupying an energy level as high as $\hbar \omega_{\text {SP }}$ above the Fermi energy. So, if one could design plasmonic devices in such a way that a large number of plasmonic particles can be packed per $\mathrm{cm}^{2}$, say for example $10^{11}$ particles per $\mathrm{cm}^{2}$, one could potentially produce as many as $10^{24}$ electrons per second per $\mathrm{cm}^{2}$ (based on the above time scale, one could create $10^{13}$ hot electrons per second, and assuming that there is at least one hot electron that has energies as high as $\hbar \omega_{\text {SP }}$ above the Fermi energy on plasmon dephasing). This is a lot of electrons, but the challenge is accessing these hot electrons for useful work, and with improvements in device engineering we hope that one could extract useful work with efficiencies rivalling conventional semiconductor-based devices. But currently, little is understood about how efficiencies depend on dynamics (the energy and momentum distribution of hot carriers), excitation intensity (and their linear and non-linear effects), pulse width, etc.

Mikhail Noginov said: In the cartoon scheme of the process, electrons are on one end and holes are on the other end. Reduction happens on one end and oxidation happens on the other end. However, at localized plasmon oscillations, positive and negative ends change with the frequency of light oscillation. Will the chemical reaction follow the change of polarity?

Martin Moskovits replied: The hot carriers produced as a result of plasmon decay are used to carry out the chemical reactions. As long as the laser is 'on', a new plasmon will be continuously excited incoherently with the previous plasmon, and hot carriers will be continually created with many hot electrons occupying energy states well beyond the Fermi energy, and it is these carriers that take part in the chemical reaction-the chemical reaction will not follow the change of polarity. But it is an intriguing question as to how the incident photon energy is distributed between the excited hot carriers and whether the relative oxidizing and reducing power of the plasmonic catalytic metal changes with time scale. We do not yet know the answer to the above. 
Kaiqiang Lin asked: In your talk, I noticed that you have changed your hot electron harvesting system from gold and silver to palladium. For the palladium system, the special thing is that it has a strong photothermal effect, which means that the local temperature could be very high. So my question is whether the photothermal effect is helping the hot electron harvesting process, or are they just competing with each other for absorption energy? How can the contributions of these two parts be distinguished? Also, what kind of plasmonic material would be more promising?

Martin Moskovits responded: These are excellent points. Plasmon-mediated chemical processes may occur as a result of: (i) the hot charge carriers resulting from plasmon decay; (ii) intense local temperatures created as a result of concentrated electromagnetic fields that enhance the chemistry; and (iii), the plasmonic system behaving as an optical antenna, thereby improving light collection by concentrating or redirecting light. All of the above processes can either individually or collectively influence the photocatalysis process, and, it is important to understand the contribution from each process. We primarily believe that the increase in quantum efficiencies observed here (Faraday Discuss., 2015, DOI: $10.1039 / \mathrm{C} 4 \mathrm{FD} 00185 \mathrm{~K}$ ) is primarily due to non-thermalized electrons, although one cannot completely eliminate thermal or local EM field enhancement effects. We place our argument based on the fact that most chemical reactions have energetics in the order of $\mathrm{eV}$, and for the process to be a wholly thermally-driven process, extremely high temperatures need to be sustained with minimal heat losses. Currently we do not have a clear-cut solution as to how one could distinguish the contributions of these two parts, although we are aware of a few studies that use local temperature probes to study plasmon-induced thermal effects.

Felix Benz said: Is it possible to use alternative materials (e.g. TiN or $\mathrm{ZrN}$ ) for emitting hot electrons? Would they be good candidates for large area applications like photocatalytic production of fuels?

Martin Moskovits responded: Certainly, in fact TiN nanoparticles have shown very good electrocatalytic activity towards the oxygen reduction reaction and have proved to be stable for the reduction of alkynes to alkenes.

Javier Aizpurua asked: In the dynamics of the electrons and holes in your antennas, there must be strong modifications to the lifetimes and to the mean free paths due to the particular configuration of the surfaces and interfaces forming the antenna. I am thinking of a different scale of distances, more connected with the roughness on the surfaces. These effects must have a tremendous impact as to how the electron makes it out from the antenna towards the catalyzer. Do you think this aspect is important in determining the efficiency? Have you checked how surface roughness, and other antenna surface effects affect the performance of your processes?

Martin Moskovits answered: Our initial experiments were performed on nanorough surfaces. ${ }^{1}$ We were able to extract hot electrons directly from the rough surfaces and the majority of the hot electrons arrived at the nanoparticles surface 
ballistically. We haven't checked our quantum efficiency as a function of surface roughness, although we thoroughly agree that this will be a fascinating study to perform.

1 V. M. Shalaev, C. Douketis, J. T. Stuckless and M. Moskovits, Phys. Rev. B, 1996, 53, 11388.

Pavel Ginzburg opened a general discussion of the paper by Mikhail Noginov by commenting: Thank you for presenting this very interesting contribution. My general question is on the theoretical interpretation of the experimentally obtained data. One of the strong assumptions, made for the fitting, is the single exponential nature of the decaying fluorophores. This assumption leads to the emergence of an additional square root of time component, if the acceptor molecule is present next to the donor. While the single exponential decay law holds for the majority of dyes in various solutions, the presence of the nearby nanostructure could make the decay process deviate from this single exponential behaviour. The decay dynamics will be dependent on the relative position and orientation of an emitting dipole relatively to a structure. My question is about the importance of those effects on the theoretical description of the FRET in the vicinity of hyperbolic nanostructured metamaterials.

Mikhail Noginov replied: This concern is, in principle, valid. However, the experimental emission kinetics of pure donors did not significantly deviate from a single exponential form. When we divided the kinetics of the donors in the presence of the acceptors by the kinetics of the donors only, we, in the first approximation, cancelled the contribution of radiative decay and non-radiative quenching (e.g. by metal) of the donors. In this way, we extracted the contribution of the donor-to-acceptor energy transfer to the emission kinetics.

Pablo de Roque asked: As far as I understood, the main claim of your paper (Faraday Discuss., 2015, DOI: 10.1039/C4FD00184B) is that, given that you have an increase of the radiative decay rate, then the efficiency of the energy transfer decreases; did I understand correctly or am I wrong? Additionally, as pointed out by a different delegate previously, I presume that your metamaterial is quite inhomogeneous at the distance scales of intermolecular distances. This means that, for example, the local fields as a function of position in the metamaterial would look different in the plane of the metamaterial to out-of-plane of it. Given that your measurement averages over a relatively large volume of the metamaterial/donor-acceptor layer, I therefore think it is bold to adventure any direct link between changes in the decay rate of emitters and the rates of energy transfer.

Mikhail Noginov responded: We have experimentally observed that the same dielectric environments that enhance the emission decay rate of donors inhibit energy transfer to acceptors. At this time, we make a statement of this experimental fact and avoid any further speculation.

Michael Flatté said: As a theorist, looking at the experimental data showing the Foerster's energy transfer, the slope that you draw on the plot in the inset of Fig. 2 in your paper, Faraday Discuss., 2015, DOI: 10.1039/C4FD00184B (slope $\approx 1 / 2$ ), for the metamaterial, appears to be consistently above the line on the left and below 
the line on the right. Is that actually a fit or is it a guide to the eye? What is the actual power law? How confident are you that this is in the square root of time regime, as the actual slope doesn't appear to support that?

Mikhail Noginov answered: The slope of the curve in the inset of Fig. 2 in our paper (Faraday Discuss., 2015, DOI: 10.1039/C4FD00184B) is $\sim 0.6$, much closer to $1 / 2$ than to 1 . Moreover, inhibition of the energy transfer on top of a metamaterial is evident from a comparison of curve 2 (emission of donors in the presence of acceptors on top of glass) and curve 5 (emission of donors in the presence of acceptors on top of a metamaterial) of Figure 2. The decay rate on top of a metamaterial is obviously slower. This conclusion does not depend on the $\sqrt{ } t$ dependence of the energy transfer term.

Jeremy Baumberg enquired: In the emission-time plot, if you look at the acceptors alone on the metamaterial, you don't have that curve on there, so what do you see for that (would it be useful to add this to the graph)? If they are also very fast, we wouldn't expect to see that much difference with the metamaterial?

Mikhail Noginov responded: The behavior of acceptors in the presence of metal is qualitatively the same as that without metal.

Francisco Rodríguez Fortuño was invited to make some remarks to open a general discussion of the paper by Olivier Martin, saying: I would like to draw attention to the possibility for an interesting optical force. Typically, optical forces are associated with gradients in the amplitude of light, however the vector character of electromagnetic fields means that light polarization can also play an important role.

Works on optical torques induced by polarization are well known. ${ }^{1}$ However, we ask whether polarization can be responsible for a mechanical net force which pushes the particle as a whole (unlike a torque). Prof. Anatoly Zayats and I, in collaboration with Prof. Nader Engheta and Prof. Alejandro Martínez, have studied the possibility of achieving a lateral force acting on a centrosymmetric particle near a surface: this force is perpendicular to the illumination direction and parallel to the surface, and it changes direction according to the polarization. The illumination can be a simple polarized plane wave with no incident field

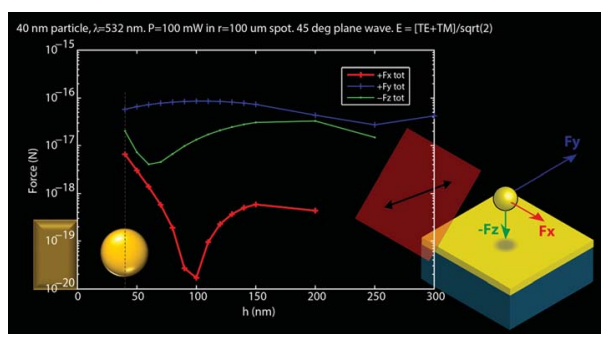

Fig. 3 The force acting on a $40 \mathrm{~nm}$ spherical gold nanoparticle suspended in water, at varying distances ' $h$ ' over a $50 \mathrm{~nm}$ thick gold film on a glass substrate, and illuminated at 45 degrees incidence by a polarized plane wave at a wavelength of $532 \mathrm{~nm}$, near the localized resonance of the particle. 
gradients. The particle need not be chiral, since the symmetry is broken by the polarization. This suggests the fascinating possibility of a very simple and switchable "nano conveyor belt" that can act on several particles simultaneously, as part of the nanofactories envisioned by Prof. Olivier Martin.

What is the origin of this lateral force? Our inspiration came from recent works on the unidirectional excitation of electromagnetic modes. By illuminating at grazing angle a particle near a metallic surface, its dipolar scattering can couple to surface plasmons that are launched unidirectionally towards the left (or right) of the particle when right-handed (or left-handed) polarization is used in the illumination. We measured this experimentally in ref. 2. A simple explanation in terms of near field interference can be found in ref. 3. The phenomenon can be understood as an example of spin-orbit coupling of light, and is possible thanks to the transverse spin of guided modes.

The existence of a polarization-switchable lateral force in such a system follows from conservation of linear momentum. The net lateral momentum of the illumination is zero, and must be conserved, which means that any lateral electromagnetic momentum carried by the asymmetric scattering of the particle (e.g. unidirectionally launched surface plasmons) must be accompanied by an equal and opposite mechanical momentum (recoil force) exerted on the particle. I think that the ability to generate equal but opposite momenta of such different natures is a fascinating possibility: we are inducing electromagnetic momentum directed to one side, and harvesting the mechanical momentum directed to the other. Since, as described above, the unidirectional excitation of electromagnetic modes is controlled by the polarization of the incident plane wave, so must the direction of the lateral recoil force be, achieving the desired switchable "conveyor belt". The concept is sufficiently general to be applicable to many different scenarios. For example, a spin-dependent recoil force is expected in every instance in which spin-orbit coupling causes a directional excitation of fields.

1 R. A. Beth, Phys. Rev., 1936, 50, 115.

2 D. O'Connor, P. Ginzburg, F. J. Rodríguez-Fortuño, G. A. Wurtz and A. V. Zayats, Nat. Commun., 2014, 5, 5327.

3 F. J. Rodríguez-Fortuño, G. Marino, P. Ginzburg, D. O'Connor, A. Martínez, G. A. Wurtz and A. V. Zayats, Science, 2013, 340, 328.

Silvan Schmid addressed Francisco Rodríguez Fortuño: The calculated force acting in the plasmonic particle seems to be very small. I am concerned that the random Brownian motion due to the photothermal heating of the particle will cancel the effect of this directional plasmonic force. As a comparison, the plasmonic forces acting on nanoparticle dimers are typically $4-5$ orders of magnitude larger.

Francisco Rodríguez Fortuño responded: Thank you for bringing up this very important practical issue. My slides showed a recent simulation result obtained on the previous day. In this numerical calculation, we used a commercial electromagnetic solver to calculate the force acting on a $40 \mathrm{~nm}$ spherical gold nanoparticle suspended in water, at varying distances ' $h$ ' over a $50 \mathrm{~nm}$ thick gold film on a glass substrate, and illuminated at 45 degrees incidence by a polarized plane wave at a wavelength of $532 \mathrm{~nm}$, near the localized resonance of the particle. The force was computed using Maxwell's stress tensor. The power density of the 
plane wave was taken equivalent to that of a $100 \mathrm{~mW}$ beam focused on a $200 \mu \mathrm{m}$ diameter spot. Directional excitation of surface plasmons accompanied by a polarization-dependent lateral force was confirmed. The specific slide is shown in the attached figure (Fig. 3). The order of magnitude of the force is around $10^{-19}$ to $10^{-17} \mathrm{~N}$. First of all, I would like to acknowledge that this force is indeed orders of magnitude below typical plasmonic forces, and we are aware that such a small force would be 'drowned' by Brownian motion. For this observation I thank you. However, I would also like to stress that the simulation shown was simply intended to provide a confirmation of the mere existence of a lateral force, as we hoped from our previous theoretical study. Therefore, I think that it is premature to judge the practical applicability of the concept on the basis of this particular simulation, with very specific parameters. Clearly there is much room for improvement in the design of a practical experiment. Our last simulations show that increasing the size of the scatterer from $40 \mathrm{~nm}$ to $80 \mathrm{~nm}$ already results in a 1 order of magnitude increase in the amplitude of the force. Also notice that we assumed a beam diameter of $200 \mu \mathrm{m}$, which in practice can be reduced, increasing the incident power density, and therefore the force. Other considerations of materials, particle shapes and wavelengths can easily be explored. Also, an important result of the simulation presented lies not in the absolute value of the force, which depends greatly on the specific parameters, but instead in the relative amplitudes between the different force components. If we compare them, we see that the lateral force is rapidly approaching the same magnitude as the pressure force when the particle gets closer to the substrate. This means that we can achieve lateral polarization-dependent forces comparable to the pressure force, and the latter have been successfully measured experimentally (for various sizes of particles and materials). In an experiment we will coat the particles and the substrate to avoid sticking between them, and use nanofluidics to place the particles in close proximity to the substrate. In addition, if we find it difficult to increase the force significantly, we can resort to a statistical analysis of the Brownian motion in order to detect an average drift caused by the lateral force. The fact that the force is lateral (perpendicular to all other forces in the system) and switchable with the incident polarization provides a method of easy experimental discrimination. Opposite average drifts should be measured for opposite polarizations. This differential measurement allows any experimental systematic drift to be compensated for. As a final remark, unidirectional excitation has been clearly visible in various experiments related to optical spin-orbit coupling (see as an example ref. 1-4), and in all these cases there is an opportunity to measure a recoil force. A particularly promising case of force measurement would be that in which the emitters are single atoms, such as in ref. 1 .

1 R. Mitsch, C. Sayrin, B. Albrecht, P. Schneeweiss and A. Rauschenbeutel, Nat. Commun., 2014, 5, 5713.

2 D. O'Connor, P. Ginzburg, F. J. Rodríguez-Fortuño, G. A. Wurtz and A. V. Zayats, Nat. Commun., 2014, 5, 5327.

3 F. J. Rodríguez-Fortuño, I. Barber-Sanz, D. Puerto, A. Griol and A. Martínez, ACS Photonics, 2014, 1, 762.

4 J. Petersen, J. Volz and A. Rauschenbeutel, Science, 2014, 346, 67.

Olivier Martin addressed Francisco Rodríguez Fortuño: I have one question and one remark to the invited comment you presented after my paper (Faraday 
Discuss., 2015, DOI: 10.1039/C4FD00224E): firstly, it seems that the optical forces you calculate are orders of magnitude smaller than the typical optical forces for the type of illumination you use (usually these forces are in the tens of pN range), maybe because the lateral effect is small, but the scattering force should be in that order of magnitude as well. Secondly, I believe that the lateral force you observe on your particle is a mere transfer of the circular polarization made possible by the feedback produced by the surface. It is similar to a (rather difficult) carom billiard shot, where one gives the ball some transverse spin (i.e. the spin axis is along the ball motion). This results in a transverse motion of the ball, equivalent to the transverse force that you observe in your calculations.

Francisco Rodríguez Fortuño replied: Thank you very much for the comment. In regard to your question, I refer to my more lengthy reply to Silvan Schmid, who raised the same issue. The force I calculated was for a given specific scenario, in which a $40 \mathrm{~nm}$ diameter gold particle is illuminated by a plane wave whose power density is equivalent to $100 \mathrm{~mW}$ on a $200 \mu \mathrm{m}$ diameter spot. The simulation was simply intended to confirm numerically the existence of the lateral force which we had predicted analytically. Is it possible that the small size of the particle and the relatively large area of illumination (resulting in a smaller power density) are the reasons for the low scattering force? In any case, it is exciting to see that the lateral $\mathrm{F}_{\mathrm{x}}$ force comes very close in magnitude to the pressure $\mathrm{F}_{\mathrm{y}}$ force (especially at small distances from the substrate), meaning that the lateral force is at least comparable with other well-known forces that have been successfully measured in the past. Regarding your insightful remark, certainly this is a good interpretation. The surface is absolutely required for this effect. The circular polarization together with the surface provide the necessary breaking of mirror symmetry that enables a lateral force. As a simple test of this, it is easy to prove using symmetry arguments that the lateral force is zero under normal illumination (incidence perpendicular to the surface). Building upon the spin discussion, in this case the illumination has a longitudinal spin (directed along the propagation direction, as is usual in circularly polarized light) which is transferred to the polarization of the particle. This rotating polarization then couples to a surface mode propagating laterally, but only in one direction, due to the transverse spin of any guided TM mode that exists in the nearby surface (with spin perpendicular to the propagation direction). This asymmetry in the excitation of modes provides the possibility of a lateral force. This 'spin interpretation' of the directional symmetry-breaking excitation of modes has been discussed in depth for experiments. ${ }^{1,2}$

1 J. Petersen, J. Volz and A. Rauschenbeutel, Science, 2014, 346, 67.

2 D. O'Connor, P. Ginzburg, F. J. Rodríguez-Fortuño, G. A. Wurtz and A. V. Zayats, Nat. Commun., 2014, 5, 5327.

Niek van Hulst commented to Francisco Rodríguez Fortuño: You show a very interesting concept, to steer forces on particles by simply switching between leftand right-handed circular polarisation. As a comment, recently the Kuipers group at AMOLF-Amsterdam showed work on sub-wavelength apertures, acting both as electric dipolar sources and as magnetic sources. ${ }^{1}$ Driving the aperture with circularly polarised light, in near field contact with a waveguide, they could show launching of light to the left or right direction depending on the direction of the 
inserted circular polarization. In that configuration, possibly forces for left-right steering might occur.

1 B. le Feber, N. Rotenberg and L. Kuipers, Nat. Commun., 2015, 6, 6695.

Francisco Rodríguez Fortuño answered: Absolutely, I agree that there is most probably a polarization-dependent force in that case. I think that, following conservation of momentum, we would expect to see a polarization-dependent "recoil force" in any experiment in which there is polarization-switchable directional emission of electromagnetic modes. Thank you for your comment and for bringing this exciting possibility to our attention.

F. Javier García de Abajo opened a general discussion of the paper by Olivier Martin: You are presenting a very inspiring concept, where optical forces are used to position nano-objects and make them interact. I am worried, however, that van der Waals forces might be comparatively larger than optical forces when the objects are in close proximity to a surface or to each other. Is this a source of concern?

Olivier Martin replied: It is true that when we started our first experiments, we were worried that van der Waals forces would be stronger than the optical forces, especially for extremely small distances between the trap and the trapped particle. Actually, the experiments showed otherwise. ${ }^{1}$ In particular, Fig. $2 \mathrm{~d}$ in ref. 1 indicated that the nanoparticle is released once the trapping laser is turned off. Later in time in that same figure, another nanoparticle makes an excursion through the antenna gap without being trapped (since the trapping laser is off), indicating that van der Waals forces will not be sufficient to trap the particle in the vicinity of the trap. One should keep in mind that all these experiments are performed in water, which screens the van der Waals interaction. Furthermore, calculations have indicated that the van der Waals force becomes significant only at extremely short distances from the surface. ${ }^{2}$ In any case, this is definitely a very interesting question to address-and possibly to utilize-for producing additional degrees of freedom in those systems.

1 W. Zhang, L. Huang, C. Santschi and O. J. F. Martin, Nano Lett., 2010, 10, 1006.

2 L. Novotny and C. Henkel, Opt. Lett., 2008, 33, 1029.

F. Javier García de Abajo asked: Optical forces have been extensively used to trap and manipulate atoms, but doing this in close proximity to a surface remains a challenge, as van der Waals forces dominate at distances below $\sim 100 \mathrm{~nm}$ for realistic light intensities. In the opposite extreme, large objects can feel much larger optical forces (e.g., a gradient force roughly proportional to their volume or the pressure force that aims solar sails in spacecraft), which are extensively used in optical tweezers and have also proved useful to steer particles down to tens of nanometers in size. ${ }^{1}$ Therefore, there seems to be a compromise between the size of the objects and their proximity to a surface. What are the size and distance ranges that you envision?

1 M. Geiselmann, R. Marty, J. Renger, F. J. García de Abajo and R. Quidant, Nano Lett., 2014, 14, 1520. 
Olivier Martin responded: Actually, the positioning of nanoscopic polarizable entities in the hot spot of a plasmonic nanostructure was already pioneered in 2010 by W. Zhang et al. ${ }^{1}$ Regarding the competition between van der Waals forces and optical forces, Novotny et al. conclude that it is only at very short distances (a few nanometres) that van der Waals can compete with optical forces. ${ }^{2}$ Actually, in all the experiments we have performed, we did not observe any disturbance caused by van der Waals forces (see e.g. the releasing experiments reported in Fig. 2d in the aforementioned 2010 Nano Letters article ${ }^{1}$ ). One should also keep in mind that most optical trapping experiments are performed in water, which strongly screens the van der Waals potential.

1 W. Zhang, L. Huang, C. Santschi and O. J. F. Martin, Nano Lett., 2010, 10, 1006.

2 L. Novotny and C. Henkel, Opt. Lett., 2008, 33, 1029.

Jeremy Baumberg remarked: In the calculation of internal strain, can you convert the forces into an actual strain from the Young's modulus? It would be interesting to know, since it is likely to be extremely small, correct?

Olivier Martin answered: As a matter of fact, the electromagnetic forces on nanostructures are too small to result in any considerable strain. For typical illuminations, the forces on plasmonic nanostructures are of the order of piconewtons. Considering a nanostructure cross section of $1000 \mathrm{~nm}^{2}$, this results in a stress of $1 \mathrm{kPa}$. Since the Young's moduli of plasmonic materials such as gold and silver are of the order of $100 \mathrm{GPa}$, the strain on the nanostructures is merely $10^{-8}$. This value is too small to be of any consequence.

Santhosh Kotni commented: As the trapping of particles or molecules in the nanojunctions strongly depends on the intensity of the electromagnetic field in the hot spots, please could you explain the distribution of electromagnetic field

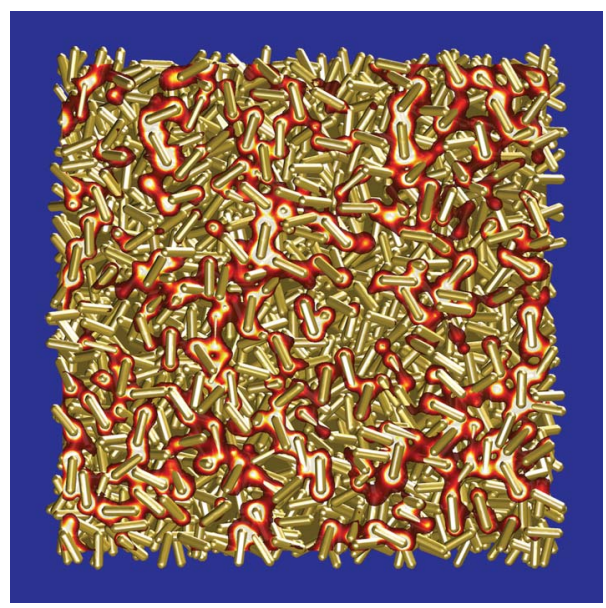

Fig. 4 Near field calculated for a distribution of 1500 gold nanorods. Reprinted with permission from D. M. Solis, J. M. Taboada, F. Obelleiro, L. M. Liz-Marzán and F. J. García de Abajo, ACS Nano, 2014, 8, 7559. Copyright 2014 American Chemical Society. 
across the hot spots. Is the field uniform across the gap, and if not, how does the field intensity distribution affect the position of the particle in the hot spots?

Olivier Martin responded: This is an interesting question, the answer to which depends a lot on the type of plasmonic structure used for trapping. In the case of a dipole antenna as studied in Fig. 3 and 4 of our paper (Faraday Discuss., 2015, DOI: $10.1039 / C 4 F D 00224 \mathrm{E}$ ), we can deduct from the charge distributions that the field is quite homogeneous within the gap of the structure, with one single hot spot, where the particle will be trapped. So we can infer one trapping position in such a geometry. This is actually confirmed by earlier studies, where we computed the trapping potential-without the presence of the particle, though-and observed one single trapping well: see e.g. Fig. 3c in ref. 1. For more complex trapping structures, the potential will be more complicated and this is certainly an issue when designing such systems. For example, in the case of the trapping hexamer shown in Fig. 6 of our Faraday Discussions paper, the trapping potential has a 6-fold symmetry, with a well-defined minimum.

1 W. Zhang, L. Huang, C. Santschi and O. J. F. Martin, Nano Lett., 2010, 10, 1006.

Jeremy Baumberg asked: Please could you comment generally on how the optical forces compare to optical heating and convection flows. Do you see any way to get around all of that realistically?

Olivier Martin replied: Forces created by convection flows or even temperature gradients can indeed dramatically disturb optical trapping experiments on plasmonic nanostructures. This is a fairly unexplored field of research and it is difficult to find quantitative data on the corresponding forces. Braun et al. have performed experiments on thermophoretic trapping of small objects, ${ }^{1}$ in a similar structure to the hexamer we discuss in our paper (Faraday Discuss., 2015, DOI: 10.1039/C4FD00224E). They have observed trapping potentials caused by thermal gradients in the order of $10 \mathrm{KT}$, which is comparable to the trapping potential caused by a plasmonic nanostructure (see e.g. Fig. $3 \mathrm{c}$ in ref. 2). In experiments we performed, we have tried to minimize thermal effects using microfluidics: a relatively shallow microfluidic channel (10-15 microns) prevents the build-up of convection flows, while the water flow through the channel provides some additional cooling to the system. Still, this is definitely a very interesting question and-possibly - the combination of thermal effects with optical trapping could provide additional degrees of freedom for manipulations at the nanoscale.

1 M. Braun and F. Cichos, ACS Nano, 2013, 7, 11200.

2 W. Zhang, L. Huang, C. Santschi and O. J. F. Martin, Nano Lett., 2010, 10, 1006.

F. Javier García de Abajo opened a general discussion of the topics raised at the meeting by saying: Advances in nanofabrication and the ability of solving Maxwell's equations in the presence of nanostructured materials have been often argued to be responsible for the explosive growth of plasmonics. Additional understanding gathered from simple analytical models has been combined with simulations for increasingly complex systems. However, there are situations in which simulations of large systems are extremely useful, for example in the determination of the position, frequency of occurrence, and distribution of 
plasmonic hotspots in realistic, random structures. I would like to emphasise that such simulations are now mastered by a handful of groups that specialise in solving the electromagnetic problem. As an example, I attach a figure extracted from Ref. 1 (Fig. 4), in which a structure formed by over 1500 gold nanorods was considered, with a random arrangement of the particles (starting from a randomly oriented nanorod that approaches the system until its distance from any other surface is $1 \mathrm{~nm}$, and repeating this process for each additional rod). The figure shows the near field, calculated with well-converged methods developed within the group led by Profs. J. M. Taboada and F. Obelleiro, and taking a few hours of simulation time in a computer cluster. In my opinion, the combination of suitable boundary element methods and acceleration procedures developed by the noted group sets a new standard for the complexity of plasmonic systems that we are currently capable of simulating within the plasmonics community.

1 D. M. Solis, J. M. Taboada, F. Obelleiro, L. M. Liz-Marzán and F. J. García de Abajo, ACS Nano, 2014, 8, 7559.

Olivier Martin commented: The results presented in the aforementioned ACS Nano paper by D. M. Solis et al. ${ }^{1}$ are truly impressive and illustrate the tremendous developments in the surface integral equation (SIE) method since the original work by A. M. Kern et al., ${ }^{2}$ its extension to periodic structures by B. Gallinet et al. ${ }^{3}$ and to the calculation of non-linear effects by J. Butet et al. ${ }^{4}$ There is a family of numerical methods, which bear similarities with the boundary equation method introduced by F. J. García de Abajo et al. ${ }^{5}$ All these techniques take advantage of the integral form of Maxwell's equation and can handle complex boundary conditions, including surfaces and stratified backgrounds. They are also particularly well-suited to simulate realistic nanostructures and provide a guide for experiments, as shown by A. M. Kern et al. in the case of fluorescence. ${ }^{6}$

1 D. M. Solis, J. M. Taboada, F. Obelleiro, L. M. Liz-Marzán and F. J. García de Abajo, ACS Nano, 2014, 8, 7559.

2 A. M. Kern and O. J. F. Martin, J. Opt. Soc. Am. A, 2009, 26, 732.

3 B. Gallinet, A. M. Kern and O. J. F. Martin, J. Opt. Soc. Am. A, 2010, 27, 2261.

4 J. Butet, K. Thyagarajan and O. J. F. Martin, Nano Lett., 2013, 13, 1787.

5. F. J. García de Abajo and A. Howie, Phys. Rev. B, 2002, 65, 115418.

6 A. M. Kern and O. J. F. Martin, Nano Lett., 2011, 11, 482.

Sang-Hyun Oh said: With new techniques to produce nanogap structures, we also face significant challenges in computational modeling. For example, using atomic layer deposition, it is now possible to make 1-2 $\mathrm{nm}$ gaps that are as long as millimeters or even centimeters in perimeter. ${ }^{1}$ From a modeling perspective, the extremely tight optical confinement in these nanogaps can give rise to an enormous mismatch in critical length scales (e.g. $1 \mathrm{~nm}$ gap vs. millimeterwavelength incoming radiation). This mismatch can quickly overcome the capabilities of conventional FDTD or FEM methodologies. Similar problems are often encountered in aerospace engineering, and advanced techniques exist to tackle such multi-scale modeling problems. One such technique-known as Hybridizable Discontinuous Galerkin (HDG) - has been applied to terahertz nanogap modeling recently, ${ }^{2}$ where the authors showed full 3D modeling of $\mathrm{THz}$ resonances in $2 \mathrm{~nm}$ gaps. I believe that such multi-scale modeling techniques 
will be useful in other problems such as sub-nanometer gaps, nonlocality, and atomic-scale materials.

1 X. Chen et al., Nat. Commun., 2013, 4, 2361.

2 H.-R. Park, X. Chen, N.-C. Nguyen, J. Peraire and S.-H. Oh, ACS Photonics, 2015, 2, 417.

Jeremy Baumberg commented: I'm interested in the comment about electron microscopy not entering here so much (EELS)-one reason is that we're in a chemistry institution. There's been some attempts to do chemistry in electron microscopy environments, but it is very difficult and you don't know that what you're seeing is useful in real systems. Thus the chemistry community are likely going to stay further away from it and work at ambient conditions. On the other side, we're also now looking at structures where atoms matter. Then electron microscopy is also not helpful really-it mostly looks at 3D arrangements of atoms down lines (or single-molecule-thick structures). It's hard to see in 3D, and especially things embedded inside structures. The really interesting thing about where the field has got in the optical domain, is that we're capable of doing better than electron microscopy now, if we only understood what the spectroscopic signatures we see tell us. We have to convince the electron microscopy community that this is really useful, that now optics tells you more then electron microscopy.

F. Javier García de Abajo remarked: Electron microscopies and spectroscopies were the techniques used in the early studies of plasmons (see, for example, ref. 1) and they have ever since remained as the tools of choice for achieving the best possible spatial resolution for mapping plasmons. I believe that they will play an equally important role in the future of the plasmonics community, as they combine sub-nanometer spatial resolution and the ability to probe optical excitations better than other existing techniques. In this respect, the advance in instrumentation is impressive, particularly with the arrival of aberration-corrected setups and improved monochromators in transmission electron microscopes (TEMs). There is really no other technique that combines the spatial and spectral resolution of electron microscopes. ${ }^{2}$ As examples of applications to atomic-scale plasmonics, I find it really impressive that one can now map plasmons trapped by a single atomic defect in graphene, ${ }^{3}$ or that the plasmonic response in metal gaps can be experimentally correlated with the morphology down to the atomic detail. ${ }^{4}$ TEMs can thus study columns of atoms, but also atomically thin specimens, and they have a good vertical resolution when looking into the bulk with well-focused beams, which surpasses that of currently available optical setups. It is difficult to beat the picometer electron wavelength with light. Environmental microscopes are also offering a way of examining samples under realistic conditions, and in this context, there are many new directions, such as using graphene to wrap the specimen. But this is only part of the ongoing effort, as scanning tunnelling microscopes, low-energy and photoemission electron microscopes and secondary-electron microscopes combined with cathodoluminescence acquisition are providing further insight. Instead of showing which community does best, it is in our interest to join efforts. As a matter of fact, cathodoluminescence (CL) is a perfect example of that, combining the best of the two worlds: electron beams and optics, spatial resolution and spectral resolution. In my opinion, this explains why more groups are using CL, and there was even a 
representation during our Faraday Discussion from a manufacturer of a new line of CL setups (delmic.com), ready to be used for nanophotonics and plasmonics. Also, there is an increasing number of TEMs equipped with optical illumination, combining ultrafast capabilities and cold-field electron emission. In summary, the plasmonics community has been and surely will be benefiting from the impressive spatial resolution of electron microscopes, while extending their techniques to involve photonic technologies, including ultrafast optics. I believe that parts of the plasmonics community are engaged in extending this effort from complementary sides, so this is an exciting time for those interested in the interaction between electrons, photons and plasmons, with lots of win-win opportunities.

1 C. J. Powell and J. B. Swan, Phys. Rev., 1959, 115, 869.

2 F. J. García de Abajo, Rev. Mod. Phys., 2010, 82, 209.

3 W. Zhou, J. Lee, J. Nanda, S. T. Pantelides, S. J. Pennycook and J.-C. Idrobo, Nat. Nanotechnol., 2012, 7, 161.

4 S. F. Tan, L. Wu, J. K. W. Yang, P. Bai, M. Bosman and C. A. Nijhuis, Science, 2014, 343, 1496. 